\title{
ENHANCEMENT AND MIGRATION OF KNOWLEDGE SCIENCE
}

\author{
Shawon S. M. Rahman, Ph.D ${ }^{1}$ and Syed V. Ahamed, Ph.D ${ }^{2}$ \\ ${ }^{1}$ Associate Professor, Dept. of Computer Science and Engineering \\ University of Hawaii-Hilo, Hilo, Hawaii, USA \\ ${ }^{2}$ Professor Emeritus, City University of New York \\ Staten Island, New York 10134, USA
}

\begin{abstract}
In this paper, we present a basis for treating, evaluating and measuring knowledge as an energy acquired by knowledge centric objects in society. The energy level acquired is indicated as their knowledge potential or KnP. Some objects get more charged in the knowledge domain and exhibit a longer and/or more intense energy trail. The Rate of knowledge acquisition, intellectual caliber and need intensity driving to acquire knowledge all play a role in the knowledge energy (Kenergy) thus acquired. Bounded by established principles of measurement, units, quantification and equations for dealing with knowledge, it becomes amenable to its own (knowledge) science with laws derived from the many disciplines and their own distinctive principles. We fall back on equations from thermodynamics, electrical engineering, fluid mechanics and transmission theory to quantify the flow-properties of knowledge. Though not completely precise and accurate at this stage of development in the science of knowledge, these disciplines offer a framework for utilizing the wealth of knowledge to stand on the shoulders of many a giant in their respective expertise. In the computational domain, symbols denote both the tangible and intangible objects and linkages handle the connectivity and updating reflecting an active and dynamic profile of knowledge. Hence, objects in the physical world and their actions become symbols and their interactions become processes in the programming domain.
\end{abstract}

\section{KEYWORDS}

Knowledge Science, Flow of Knowledge, Velocity of Knowledge, Social Sciences.

\section{INTRODUCTION}

Knowledge science (KS) has been an evolving field. Knowledge has been studied for millennia from religious and spiritual (Aryan, Hindu, Greek, Judaic, Biblical, Islamic, etc.), perspectives. More recently knowledge has also been seriously studied from scientific (management, medical, educational, statistical, etc.) perspectives. However, since the beginning of this century attempted to study knowledge from a quantitative perspective have appeared [1,2]. As far back as 1990, for bus and Fakenhainer [3] have provided a framework of qualitative physics for organizing and using quantitative knowledge and provided three numerical simulations. However, the results ignore individual differences, human adaptation towards new and derived knowledge, dynamic social setting of individuals, interactive cyclic process between human beings and miss the major factor of social and human considerations in making knowledge a unified science and a firm discipline of self, mind and society. Numerous magazines have gained a foothold in Great Britain $[4,5]$. The interest in knowledge science appeared to have peaked in 2008 when BBC knowledge Magazine was chosen as one of the top ten magazines launched by Library Journal [6].

In certain practical situations, the quantitative knowledge is indispensable and crucial. For instance, the medical field is dominated by laboratory test and the quantification of the biological 
data[41][39][43].This data (measured, derived, or synthesized) is essential for diagnosis and for the remedy of diseases and ailments. Most doctors use quantified medical data and its associated science to diagnose the patient ailments and prescribe medication. Medical application programs [39][43].in conjunction with communication networks offer the medical community procedures, cures and remedies. Most of these applications provide quantitative information. As the modern society is becoming more dependent on digital devices, networks and data processing, the impact of KS in its quantitative form is becoming increasingly important. As another instance, modern corporations are more and more involved with accounting, inventory control and decisionsupport-systems. In order to navigate the human science of management, KS is an invaluable tool to balance finances, inventory and make appropriate decisions. Other applications (such as weather prediction, tsunami warning systems, geological and seismic studies are also dependent on quantitative knowledge in data.

The topics underlying KS are vast, interdisciplinary and innumerable and the foundations of expertise are not firmly established in any one specific discipline. For this reason, the contributors are few and diversified leading to the absence of knowledge science as a discipline in its own right. However, the situation is more promising during this decade due to the availability of more knowledge bases (KBs), greater human expertise, wider and more far reaching networks and hand held devices.

The demise of BBC Knowledge Magazine in 2012 [5][17],(even though the web page is still active) is indicative of the lack of cohesion and synergy in numerous discipline to make KS an area of consorted research and expertise. However, we do not believe that KS is going to disappear from the domain of sciences. Instead it needs to draw its root from numerous well established sciences. In this paper, we allude to the hard sciences (physics, electrical engineering, fluid mechanics, magneto-hydro dynamics, electric and magnetic field distribution), software sciences (application, software, and higher-level programming, microprogramming, nano-programming) to bridge the gap into social sciences (psychology, economic basis of human behavior, social sciences). The root for the since of knowledge have spread and its flowers have blossomed in many cultures and societies in harmony as the plants in the diversified geographical areas of the world[18][19].

\section{The NeEd To KNOW AND NeEd To LEARN}

Knowledge being an essential ingredient of life itself, evolution and knowledge are symbiotic as much as quantification and mathematics are symbiotic. Knowledge has had a profound impact on how human have learned to behave thus altering the mode of behavior to survive. Knowledge, behavior and survival[39][43] form a tightly knit triad of continued existence. This chain is well founded and deeply rooted. This continuum of the cyclic processes has enhanced and enriched the total energy of knowledge or their kenergy, thus accelerating the evolution of some species over that of others. Mankind has been the prime beneficiary and the accompanying creativity has further accelerated the evolutionary patterns leading into the computer, information and knowledge ages for most societies[23].

Individual actions and acceptable social behavior have also been intertwined since historic times. Individual actions form the strands in the fabric of social behavior. Science of knowledge needs the reinforcement of social science and vice versa[27].Being anchored deep in the personalities of interacting individuals, behaviorism in social sciences relies heavily on motivation and needs of human beings. The gratification of such needs leads to behavior of any particular human being in any particular society. 
From a modern perspective Maslow has offered a five layer need hierarchy [7]. Ahamed has suggested two additional layers at the top of Maslow's five-level hierarchy to include the impact of information age and Internet. The search in the Internet knowledge space and higher intellectual needs are accommodated in these sixth and seventh levels [8][11] of the enhanced need triangle. The cohesion between needs stratified in five or seven layers and behavior is strong and subtle [9][16].

The stratification of the layers and the principles of social psychology ${ }^{1}$ [7] thus become a series of programmable computational steps [10]. Styles, traumas, whims and fancies are generally transient but the social fabric of most cultures has a longer life. Being alive and well founded in the needs of all humans, it changes gradually and continuously, but it is governed by the social norms and ethics [10]. Having a deep foundation in being congenial and benevolent, they are adaptive to changing whims and fancies do not stray too far, nor too long to become outcast destructive to humankind [21][34].

Like most scientific objects, the mind retains the energy of knowledge. In Newtonian physics, moving objects designate the kinetic energy ${ }^{2}$ as $\frac{1}{2} \mathrm{mv}^{2}$; in electrical engineering ${ }^{3}$, the capacitors and inductors retain the electrical energy as $\frac{1}{2} \mathrm{CV}^{2}$, and as $\frac{1}{2} \mathrm{LI}^{2}$. Other examples also exist. In the knowledge field, the measure of the knowledge energy (or kenergy) is measured in a similar fashion. The retained kenergy is proportional to the square of knowledge potential or $\mathrm{KnP}$ and a knowledge attribute or KA of the knowledge centric object (KCO) [39][43].

Kenergy is initially symbolized as kenergy and computed as $\left(\frac{1}{2}\right.$.KA. $\left.(\mathrm{KnP})^{2}\right)$ and is equal to the work done by the particular KCO in attaining a knowledgepotential of KnP. The energy attribute can change over the life time of $\mathrm{KCO}$ and $\mathrm{KnP}$ can dissipate, degenerate, decompose, etc. for the worse or it can invigorate, reorganize, recompose, etc. for the better.

Most KCOs lose energy exponentially over time ${ }^{4}$. Knowledge though abstract and intangible has structure, connectivity and become quantifiable in terms of two basic attributes, the humanist property of a noun object and the KnP achieved by the object. In the framework of these two basic and quantifiable attributes, the acquired knowledge energy becomes quantification. These invisible lineages and linkages to other bodies of knowledge, communicate the value and validity of knowledge in the particular society where it exists. Knowledge also exhibits dynamic features such as flow and velocity since it can migrate, expand and/or shrink. This flow and velocity have both directionality and measures thus offering the discipline of fluid mechanics to quantify one aspect in the flow of knowledge. Although the assumption of conservation of energy well-founded in fluid mechanics is not applicable in the knowledge domain, the mathematical principles embedded in generalized nonlinear Bernoulli equations may offer a good basis to import the concepts into the knowledge domain. To this extent, the abstract nature of knowledge is akin to the nature good-will and/or virtue that do not diminish when they are shared.

1 as defined by Baron, Byrne \&Suls (1989) in [4] as "the scientific field that seeks to understand the nature and causes of individual behavior in social situations".

2 where $\mathrm{m}$, is the massis the moving object dependent mass and $\mathrm{v}$ is the velocity.

3 where $\mathrm{C}$ and $\mathrm{L}$ are electrical object dependent capacitance and inductance respectively. The voltage $\mathrm{V}$ across the capacitor and the currentin the inductor coil respectively, determine the energy stored in the capacitor, and in the inductive coil respectively.

4 The human tendencies to forget over shorter time durations and some aspects of senility over longer lifetime are both suggested. 
Uncertainty becomes a key ingredient in the science of knowledge since it encompasses human beings and mankind per se. We start by asserting that every human being is unique and distinctive and these individualist traits become parameters in equations within knowledge science(s). However, statisticians have developed strategies and techniques [12] to deal with uncertainty. More than being just strategies, they are incorporated as managerial tools $[12,13]$ in Management Science and used repeatedly to maximize profits, minimize risks, mini-max and maxi-min, etc. Such approaches are deployed in most business communities in seeking their specific goals.

\section{EnhanCEMEnt And Migration Of KnOWLEDGe}

In the digital age[22][18] of the last few decades, the role of computers has become progressively intense. In line with the tradition of computer architecture, binary data is grouped as nibbles, bytes, words or any form of data structure and represented as the second node in a trail of knowledge depicted in Figure 1 and discussed in Ref [14]. Operation codes (opc's) or a series of opc's process data [15] structures and become programmable steps in solving a variety of scientific, business or information based problems. There are seven nodes in Figure 1. The pattern of movement from any node (i) to the next node $(i+1)$ can be generalized. There is considerable similarity in the approach in the progression of knowledge and science, in building one computer platform after an earlier one, or even in introducing one computer language[29][31] after an earlier version (e.g., FORTRAN V after FORTRAN IV, C+ after C, C++ after C+).

When sufficient expertise is gathered in processing at each of the seven nodes (B through E), under the current rules of compilation, a more generic, powerful, and encompassing format is conceived or invented for the nodes I, K, C, W, and E. Backward compatibility is generally adhered and sometimes abandoned in light of a breakthrough process, technology or invention. In applying these guidelines to machines for processing information, knowledge, concepts, wisdom, and ethical/social values, the generic migratory path from any node (i) to next node $(i+1)$ can be extrapolated[24][26].

The role of human beings for content processing becomes evident and assumes a role similar to the role of opc's on operands. From a macroscopic perspective, the contents of the databases at each of the nodes become the operands and the human control by using the appropriate programs or applications becomes the opc's. Globally, the human beings retain all the control by providing the access to the correct data and content bases and again by choosing the most appropriate pro Grams/applications to Process the contents.

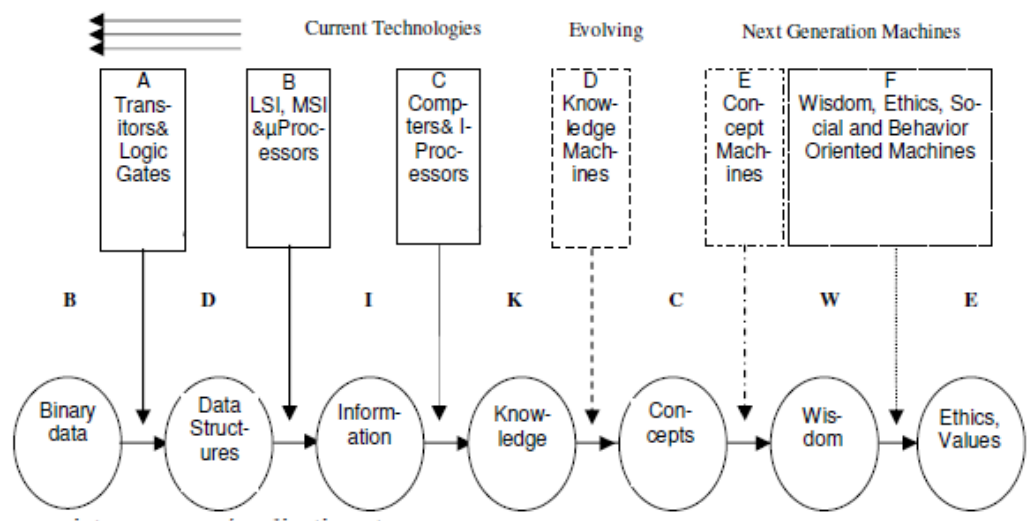

Figure 1Successive processing of binary data in computer systems leads to key concepts, wisdom, ethical and social values in any given society. Seven nodes are shown with reality of digital data that flows through the networks on the left side to ethicsand values on the right side. Microscopic functions on the left side are cumulated to achieve major functions on the right side. Currently the functions on data structures and information form a basis of programmable steps in solving scientific, business or information problems. 
The platform for the next-generation computers to process information, knowledge, concepts, or wisdom starts at platforms ( $\mathrm{i}=2,3,4$, and 5) respectively in Figure 1 . The content (full lines) comes from all the databases of prior domains and from all sources including Internet, WWW, knowledge bases, broadband and digital networks, and library databases. It is important that the content satisfy the processing capability of content at that particular node[39][43].

\section{MOVEMENT OF KNOWLEDGe In SOCIETY}

A structure for the upward migration of knowledge is shown in Figure 2. During the early days of evolution of knowledge at the lowest (seventh) level of the pyramid, structure and functionality were emphasized. In the Internet age, flow analysis of data and information is feasible and practiced by most Federal and State government agencies. Police and Intelligence agencies practice this to keep surveillance records on ordinary citizens, suspects and criminals. The data-flow records are available to most IP servers and to their personnel. The social and ethical aspects, though questionable are also in use for spam, unsolicited mail and generating junk information on theInternet at the lowest levels of this knowledge pyramid.

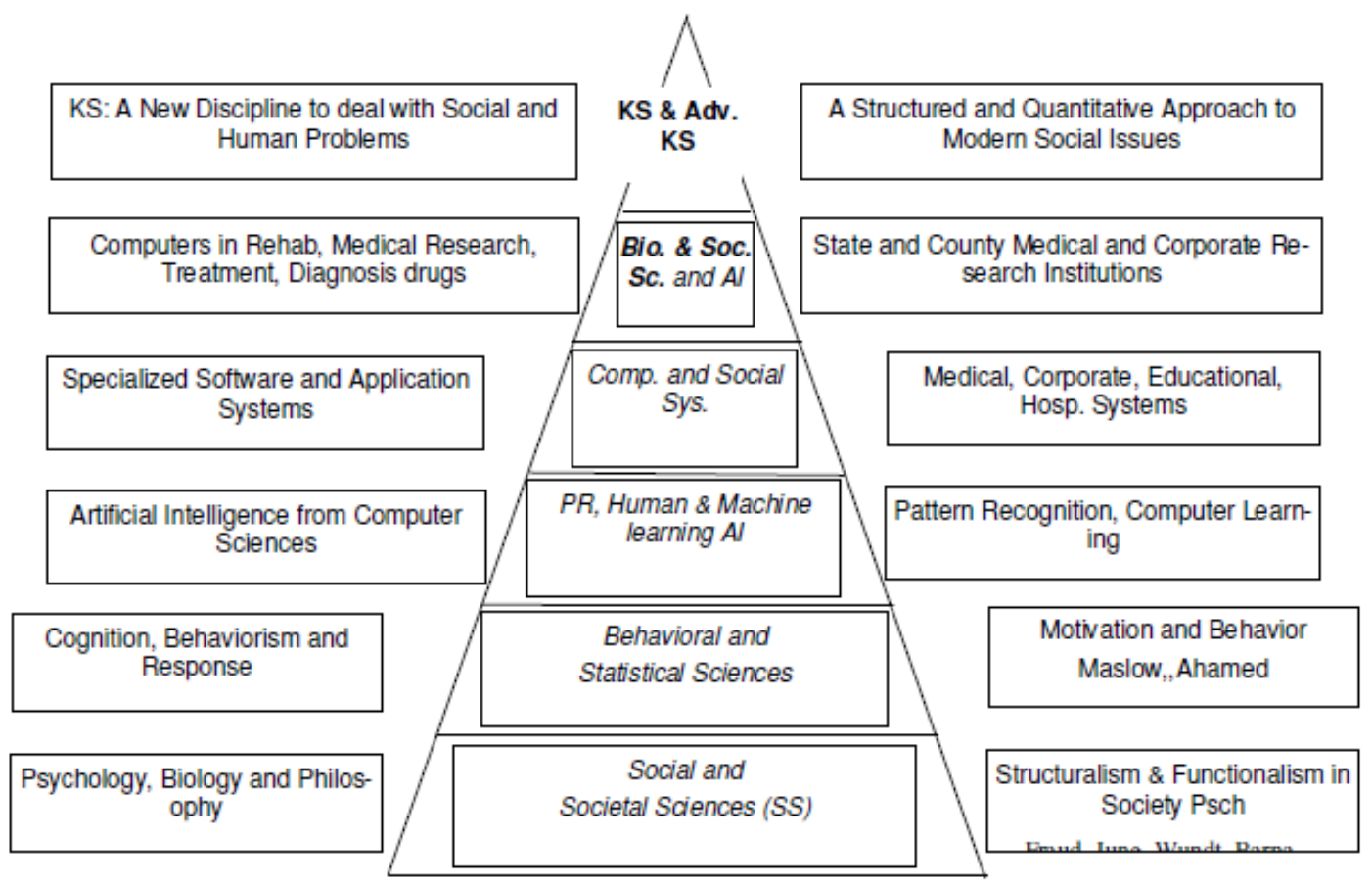

Figure 2 Pyramid of Knowledge Science for the upward migration of knowledge. During the times of the Greek philosophers at the lowest (seventh) level of the pyramid, structuralism \& functionalism were emphasized. Social sciences though not apparent still formed the basis of early learning and communication of knowledge from one generation to the next.

Unfortunately, spammer have access to the user preferences, habits and machine learning algorithms that makes the targeted marketing more annoying than ever before. Such issues can be most easily curtailed by limiting the access to user and customer data bases by encryption or indirect addressing this preventing spammers and junkies to abuse information.

At the top of the pyramid shown in Figure 2, it is possible to build a social machine to satisfy customized and personalized user needs. When the access is authorized by the user and by the responsible and licensed intermediate agencies of the Federal and State governments, the data and socially processed information can offer unique and significant results to the members in the so- 
ciety [32][33].For example, hospital with certain specific staff and facilities can provide focused information and suggestions to patients and the sick based on the patient data bases and financial history and records[25][28].Marketing and dating agencies attempt to reach their most likely customers in order to generate revenues for themselves. There is no social value in these spam messages. The social value is generated at the third and fourth levels of the pyramid of Figure $2[9][41]$

\section{CURRENT Societies ANd INFORMATION Age}

The rapid feedback and circulation of "significant, truthful and socially valuable information" is a catalyst in the explosive growth of a progressive knowledge-age on the one hand, yet one the other, a similar cycle of "junk, deceptive and socially insignificant information" is (has become) a catalyst of recessive knowledge-age by wasting users time and resources. Knowledge based on purely human deduction and inference evolves slowly and the positive or negative directions are not easily discernible. Digital encoding and computer security have also evolved slowly but the direction has been (mostly) beneficial to the society. These scientific disciplines, unlike gossip and hearsay, do not have emotional appeal to the public and are thus generally propagated in a positive direction. The inclusion of computing systems to accelerate the evolution of any discipline is evident from VLSI chip design to the design of worldwide networks.

The stagnancy in the design and architecture of social computers is evident. Human problems and issues are more prevalent and pressing over longer duration. In a sense, even the elementary practice of medicine (such as billing and counseling) had suffered similar drawbacks during 1980s and early 1990s while other disciplines (such as design of VLSI and of communication systems) were experiencing exponential rates of growth [37][42].

Figure 3 depicts a pyramid of knowledge for the growth of adjoining discipline for the next generation machines such as concept, wisdom and social machines to follow a firm structure of knowledge machines shown at Node 4 in Figure 1. Human inputs and skills are also shown to be able to design personalized social machines capable of solving interpersonal and inter-social problems. The hierarchy in Figure 3 is generic and can be used in any area of expertise such as mathematics or physics and can be used in most circumstances such as to resolve dissertation issues of a given doctoral student.

This continuum of the cyclic processes has enhanced and enriched the total energy of knowledge thus accelerating the evolution of some species over that of others. Mankind has been the prime beneficiary and the accompanying creativity has further accelerated the evolutionary patterns of learning and adaptation leading into the computer, information and knowledge ages for most societies. Significant results have been harnessed in scientific and business realms. Such strides are also feasible in the social domain 


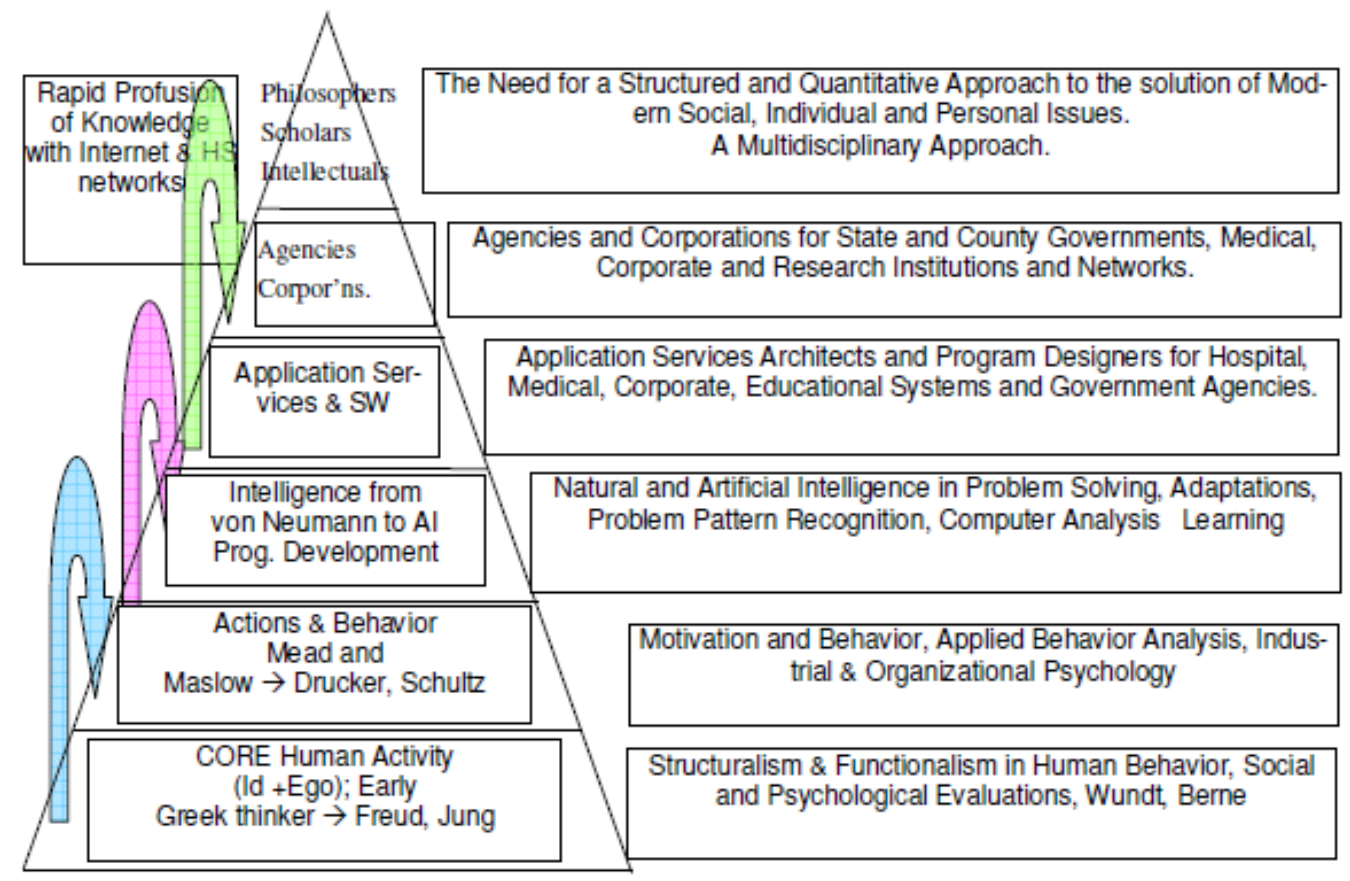

Figure 3. The need for an interdisciplinary platform of science for the systematic and cogent development of knowledge science (KS) is illustrated to include the significant contribution in knowledge domain. Solution of Social, Individual and Personal problems in the modern society will thus be resolvable by KS based on scientific basis.

\section{Social Perspective On Enhancement Of KnOWledge}

This major cycle of current knowledge (K), behavior (B), learning (L), adaptation (A), survival (S), enhanced knowledge (EK); is depicted in Figure 4. Denoted as $(\mathrm{K} \rightarrow \mathrm{B} \rightarrow \mathrm{L} \rightarrow \mathrm{A} \rightarrow \mathrm{S} \rightarrow \mathrm{EK})$, this cycle of global social progress is well founded and deeply root. Acceleration of social and cultural growth (or its decay) is the ultimate result of the high-speed data networks, ATM switches and the TCP/IP protocol[31][38].Human skills and knowledge tools have evolved more successfully in selected segments of the population around the globe, thus causing a knowledge divide between the "know-hows" and the "know-nots". This knowledge divide has acquired unprecedented momentum with new social problems between the knowledge-elite and the knowledge-impoverished. Different patterns of intellectual behavior and conduct arise and likely to become more severe. After all, wars and Mafia were a way of life till a sense of justice prevailed around the globe[35][36]. Yet, isolated families practice cruelty and violence. Perhaps social workers equipped with pocket-sized social machines may help resolve such issues as much as a thermometer or a blood pressure machine helps a visiting nurse. 


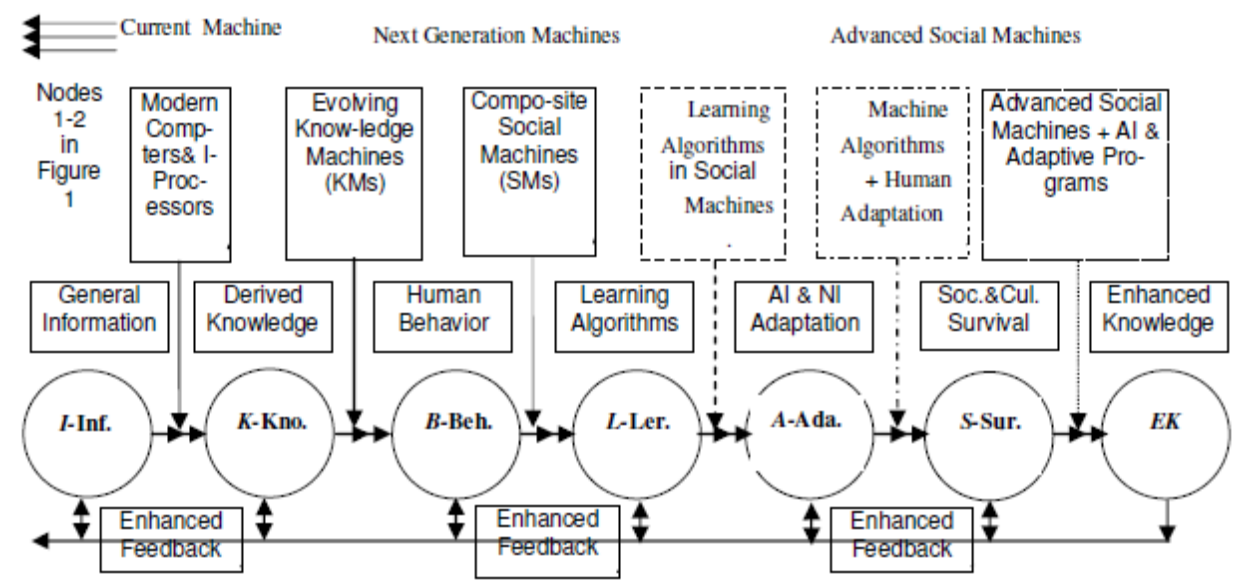

Figure 4 Continued and accelerated growth of machines, human skills and knowledge era in the current decades is indicated by the influence of information (inf.), knowledge (Kno.), Behavior (Beh.), Learning (Len.), Adaptation (Ada.), Survival (Sur.) in that particular society or culture, and finally of Enhanced Knowledge (EK). The close synergy

Unfortunately, the self-interest that is common to both segments offers the tools of Mafia to the unjust and a tainted sense of fairness to the elite. Behavior assumes a mode of cruelty for the know-nots (like the tribal kingpins in remote areas) or a mode of deception (like most of the politicians) in western democracies[40][42].

The synergy of human skills in the knowledge era is currently quantifiable by the information (inf.), knowledge (kno.), Behavior (Beh.), Learning (Ler.), Adaptation (Ada.), Survival (Sur.) in that particular society or culture, and finally of Enhanced Knowledge (EK). The role of Artificial Intelligence within Natural Intelligence makes social machines as significant to the users as automobiles are to the public. Computer and network literacy becomes unimportant to solve most problems of loneliness, aging and social/cultural adjustments

\section{CONCLUSION}

In this paper we have presented the precepts of the evolving science of knowledge intertwined with social and computer sciences. These precepts have been informally deployed in the continued evolution of knowledge coupled with behavior, learning, adaptation and survival of species over a long time. The role and influence of computer sciences and diffusion of data, information and knowledge over the Internet have been more recent but radical and dramatic. This paper presents an integrated perspective leading to the design and development of social machines to solve age-long social problems by using the technological strides during the computer and networks times of the recently passed decades. A similar situation existed during the days of Charles Babbage while he used his primitive (1820's) "Difference Engine" and his primitive (1830's) "Analytical Engine" together to generate the "Mathematical Tables" that were manually generated before his "Babbage" machines.

Nearly two centuries have elapsed before the current generation of multidisciplinary scientists can build a Hand held Tablet of "Social Tables" duly accurate and individually tailored to the user by imaging the user personality attributes into the global knowledge banks. Such "social tables" should attempt to improve the accuracy and precision of solutions to most of the human needs and problems as much as the Babbage machines contributed to the accuracy and precision of the "Mathematical Tables" of the past era. Knowledge sciences in numerous disciplines would offer a generic platform for the design and architecture of such generic social machines of the 2020's. 
International Journal of Computer Networks \& Communications (IJCNC) Vol.9, No.6, November 2017

\section{REFERENCES}

[1] "Quantified self: The Algorithm of Life", http://www.prospectmagazine.co.uk/arts-andbooks/quantified-self-the-algorithm-of-life, web page accessed September 05, 2017.

[2] "Knowledge through Numbers, Welcome to a Quantified Lifestyle", http://quantifiedselfboston.org/, web page accessed September 05, 2017

[3] Forbus, K. D., and Falkenhainer, B., "An Integration of Qualitative and Quantita-tive Knowledge", AAAI-90 Proceedings, AAAI-90 Proceedings, 1990, pp. 380-387. Also available at qrg.northwestern.edu/papers/Files/ For-bus_Falkenhainer90.pdf.

[4] “Top 10 of Everything”, BBC Knowledge - December 2014, www.bbcknowledgemagazine, web page accessed September 05, 2017.

[5] “BBC Focus”, http://en.wikipedia.org/wiki/BBC_Knowledge_(magazine), web page accessed September 05, 2017.

[6] "Ten new launches buck the downturn", Library Journal, May 1, 2009.

[7] Maslow, A. H. (1970b), Religions, values, and peak experiences. New York: Penguin, (originally published in 1964. Abraham H. Maslow has written 20 books and significantly contributed to numerous fields in sociology, behavior, motivation, and social psychology.

[8] Ahamed, S. V. (2005) "An enhanced need pyramid for the information age hu-man being", in Proceedings of the Fifth Hawaii International Conference, Fifth International Conference on Business, Hawaii, May 26-29, 2005.

[9] Ahamed, S. V. (2005 a) "An enhanced need pyramid of the information age human being, paper presented at the International Society of Political Psycholo-gy”, (ISSP) 2005 Scientific Meeting, Toronto, July 3-6, 2005.

[10] Baron, R.A., Byrne, D. E., and Suls, J. M., (1989), Exploring Social Psychology, Allyn and Bacon, 3rd Edition 1989.

[11] Ahamed, S. V. (2007) Intelligent Internet Knowledge Networks, Wiley (2007), Chapter 9, pp 241265.

[12] Deaux, K, and Synder, M., "Goal-driven cognition and functional behavior: The fundamentalmotives framework", Current Directions in Psychological Sci-ence, 19(1), 63-67.

[13] Taha, H.A.(2010), Operations Research: An Introduction (2010), Prentice Hall, 9th Ed., 2010.

[14] Drucker, P. F. (2006), The Effective Executive: The Definitive Guide to Getting the Right Things Done, Harper Business Essentials, (2006); also see, Drucker, P. F., Management Challenges for the 21st Century, Harper Business; 1st edition (June 26, 2001).

[15] Ahamed, S. V. (2007), Intelligent Internet Knowledge Machines: Processing of Concepts and Wisdom, Wiley, 2007. See Chapter 15, "Looking Ahead: Social and Ethical Implications" (pp. 444-582) and Section 15.1 for the seven node (BDIKCWE) Knowledge Trail and node transition analysis.

[16] http://www.teach-ict.com/as_as_computing/ocr/H447/F453/3_3_8/features/miniweb/pg4. htm; web page accessed on September 05, 2017.

[17] Loukaka, Alain and Rahman, Shawon; "Discovering New Cyber Protection Approaches From a Security Professional Prospective"; International Journal of Computer Networks \& Communications (IJCNC) Vol.9, No.4, July 2017.

[18] Rader, A., Marc and Rahman, Syed (Shawon); "Exploring Historical and Emerging Phishing Tech niques and Mitigating the Associated Security Risks"; International Journal of Network Security \& Its Applications (IJNSA), Vol.5, No.4, July 2013.

[19] Opala, John, Omondi and Rahman, Syed (Shawon);"Corporate Role in Protecting Consumers from the Risk of Identify theft "; International Journal of Computer Networks \& Communications (IJCNC), Vol.5, No.5, September 2013. 
[20] Todd, S., Margie and Rahman, Syed (Shawon);“ Complete Network Security Protection for SME's Within Limited Resources"; International Journal of Computer Networks \& Communications (IJCNC), Vol.5, No.6, November 2013.

[21] DeVoe, Charles and Rahman, Syed (Shawon); "Incident Response Plan for Small to Medium Sized Hospital"; International Journal of Network Security \& Its Applications (IJNSA), Vol.5, No.2, March 2013.

[22] Rahman, Syed (Shawon) and Lackey, Robert; "E-Commerce Systems Security for Small Businesses"; International Journal of Network Security \& Its Applications (IJNSA), Vol.5, No.2, March 2013.

[23] Henderson, James and Rahman, Syed (Shawon); " Working Virtually and Challenges that must be overcome in today's Economic Downturn"; International Journal of Managing Information Technology (IJMIT); ISSN : 0975-5586 (Online) ;0975-5926 (Print).

[24] Dreelin, S., Gregory and Rahman, Syed (Shawon);“ Enterprise Security Risk Plan for Small Business"; International Journal of Computer Networks \& Communications (IJCNC), ISSN : 0974 - 9322 [Online] ; 0975- 2293 [Print]).

[25] Donahue, Kimmarie and Rahman, Syed (Shawon); "Healthcare IT: Is your Information at Risk?"; International Journal of Network Security \& Its Applications (IJNSA), Vol.4, No.5, September 2012.

[26] Rice, Lee and Rahman, Syed (Shawon); "Non-Profit Organizations' need to Address Security for Effective Government Contracting"; International Journal of Network Security \& Its Applications (IJNSA), Vol.4, No.4, July 2012.

[27] Neal, David and Rahman, Syed (Shawon); "Video Surveillance in the Cloud?"; The InternationalJournal of Cryptography and Information Security (IJCIS), Vol.2, No.3, September 2012.

[28] Lai, Robert and Rahman, Syed (Shawon); "Analytic of China Cyberattack"; The International Journal of Multimedia \& Its Applications (IJMA), June 2012, Volume 4, Number 3.

[29] Halton, Michael and Rahman, Syed (Shawon); "The Top 10 Best Cloud-Security Practices in NextGeneration Networking"; International Journal of Communication Networks and Distributed Systems (IJCNDS); Special Issue on: "Recent Advances in Next-Generation and Resource-Constrained Converged Networks", Vol. 8, Nos. 1/2, 2012, Pages:70-84.

[30] Mohr, Stephen and Rahman, Syed (Shawon); "IT Security Issues within the Video Game Industry"; International Journal of Computer Science \& Information Technology (IJCSIT), Vol. 3, No 5, Oct 2011.

[31] Dees, Kyle and Rahman, Syed (Shawon); "Enhancing Infrastructure Security in Real Estate"; International Journal of Computer Networks \& Communications (IJCNC), Vol.3, No.6, November 2011

[32] Hood, David and Rahman, Syed (Shawon); "IT Security Plan for Flight Simulation Program”; International Journal of Computer Science, Engineering and Applications (IJCSEA), Vol.1, No.5, October 2011.

[33] Schuett, Maria and Rahman, Syed (Shawon); "Information Security Synthesis in Online Universities"; International Journal of Network Security \& Its Applications (IJNSA), Vol.3, No.5, Sep 2011.

[34] Jungck, Kathleen and Rahman, Syed (Shawon); " Cloud Computing Avoids Downfall of Application Service Providers"; International Journal of Information Technology Convergence and services (IJITCS), Vol.1, No.3, June 2011.

[35] Slaughter, Jason and Rahman, Syed (Shawon); " Information Security Plan for Flight Simulator Applications"; International Journal of Computer Science \& Information Technology (IJCSIT), Vol. 3, No 3, June 2011.

[36] Benson, Karen and Rahman, Syed (Shawon); "Security Risks in Mechanical Engineering Industries", International Journal of Computer Science and Engineering Survey (IJCSES), Vol.2, No.3, August 2011.

[37] Bisong, Anthony and Rahman, Syed (Shawon); "An Overview of the Security Concerns in Enterprise Cloud Computing "; International journal of Network Security \& Its Applications (IJNSA), Vol.3, No.1, January 2011. 
[38] Mullikin, Arwen and Rahman, Syed (Shawon); "The Ethical Dilemma of the USA Government Wiretapping"; International Journal of Managing Information Technology (IJMIT); Vol.2, No.4, November 2010 .

[39] Ahamed, Syed and Rahman, Syed (Shawon); "Architecture and Design of Medical Processor Units for Medical Networks"; International journal of Computer Networks \& Communications (IJCNC), Vol.2, No.6, November 2010.

[40] Rahman, Syed (Shawon) and Donahue, Shannon; "Convergence of Corporate and Information Security"; International Journal of Computer Science and Information Security (IJCSIS), Vol. 7, No. 1, 2010.

[41] Rahman, Syed (Shawon) and Ahamed, Syed; "Intelligent Network Applications for Medical Systems"; The Second International Workshop on Ubiquitous Computing (UbiC-2010),(In conjunction with WiMo 2010) - June 26- 28, 2010, Ankara, Turkey.

[42] Rahman, Syed (Shawon); Ahamed, Syed, and Erdogan, Sevki; "IT Security Assessment for Interdisciplinary Research"; The First International workshop on Communications Security \& Information Assurance (CSIA) (In Conjunction with WiMo - 2010) June 26-28, 2010, Ankara, Turkey.

[43] Ahamed, Syed and Rahman, Syed(Shawon); "Design Constructs of a Knowledge Machine"; 2010 IEEE International Conference on Systems, Man, and Cybernetics (SMC2010); 10-13, October 2010, Istanbul, TURKEY.

\section{AUTHORS}

Dr. Shawon Rahman: Dr. ShawonS. M. Rahman is an Associate Professor in the Department of Computer Science and Engineering at the University of Hawaii-Hilo, USA. Dr. Rahman's research interests includeInformation Assurance and Security, Software Engineering education, Software Testing \& QA, Cloud Computing, Mobile Application Development, and Web Accessibility. He has earned his Ph.D. in Software Engineering and MS in Computer Science from the North Dakota State University, Fargo, ND. He has published over 100 peer-reviewed articles and is a member of many professional organizations including IEEE, ACM, ASEE, ASQ, ISACA, and UPE.

Dr. Syed V. Ahamed: Dr. Syed V. Ahamed is a Professor Emeritus at the City University of New YorkStaten Island, New York, NJ. Dr. Ahamed obtained his Ph.D. from VictoriaUniversity of Manchester in the United Kingdom. Dr. Ahamed alsoholds the Doctor of Science (D.Sc.) degree from Victoria University ofManchester as well as an M.B.A. in economics from New YorkUniversity.Dr. Ahamed, a Fellow of IEEE, has a broad spectrum of teaching and research interests including,Intelligent Sensing Systems, Computer Architecture, CPU and KPU Designs, Telecommunications,Intelligent Networks, Knowledge Systems, Management In-

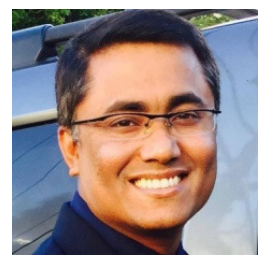
formation Systems, and Economics. He hasreceived many European and US patents and published many books and hundreds of articles. 